\title{
COVID-19-related Mucormycotic Osteomyelitis of the Maxilla: A Case Report of Findings, Surgical Management and Post-surgical Rehabilitation
}

\author{
Yaseer Arafat ${ }^{1}$, Yenugula Naren Kumar ${ }^{2}$, Kamini Bharani ${ }^{3}$, Thodur M Balaji ${ }^{4}$, Saranya Varadarajan ${ }^{5}$, Thirumal Raj $^{6}$, \\ Shankargouda Patil ${ }^{7}$
}

\begin{abstract}
Aim and objective: To report a case of surgical management and postsurgical rehabilitation COVID-19-related mucormycotic osteomyelitis of the maxilla.

Background: Patients with the SARS-CoV-2 infection, also termed the COVID-19 illness, have been found to be afflicted with other associated bacterial and fungal diseases which have been termed as co-infections. In this regard, mucormycosis, a fungal infection, has been found to occur in these patients especially with a lowered immune response and has been found to cause osteomyelitis of the jaw bones.

Case description: We present a case of mucormycosis in a COVID-19-affected patient that occurred in August 2020 denoted the first wave of disease in India, causing extensive osteomyelitis of the left maxilla. The patient presented clinically with odontalgia and tooth mobility in the upper anterior region. Clinical examination revealed the presence of abscesses in the attached gingiva in relation to tooth numbers 13,12, 11, $22,23,24$ and 25 . A deep horizontal cleft was observed in the mucogingival junction in relation to 23 discharging pus. Computed tomography (CT) imaging revealed a diffuse radiolucency extending from the alveolar ridge of the upper anterior teeth to the left maxillary sinus, breaking the floor of the sinus. We have performed extensive medical and surgical management of this patient including postoperative prosthetic rehabilitation which is documented in the present case report.

Clinical significance: Our observation of maxillary osteomyelitis secondary to mucormycosis in the first COVID wave in India makes our case extremely rare and important as this highlights that COVID-19 complications were significant but under-evaluated in the first wave of the disease. Keywords: COVID-19, Maxillary osteomyelitis, Mucormycosis.

World Journal of Dentistry (2021): 10.5005/jp-journals-10015-1864
\end{abstract}

\section{INTRODUCTION}

The whole world today is battling the COVID-19 illness which is a deadly disease scourge that has spread its tentacles and affected human populations in both developing and developed nations. The disease has been upgraded as a pandemic by the World Health Organization ${ }^{1}$ and is caused by the SARS-CoV-2 virus, characterized by the presence of a spike glycoprotein, ${ }^{2}$ hence also termed by the layman as coronavirus infection. The main cause of death in the SARS-CoV-2 infection is due to acute respiratory distress caused by viral infiltration into the lung tissues that causes viral pneumonia. ${ }^{3}$ In India, COVID-19 illness has been affecting human subjects since March $2020 .{ }^{4}$ Despite the introduction of vaccination regimens which are operating in a steady fashion from January 2021, the disease has not left mankind and the current phase in India has been termed second wave of the disease affliction. ${ }^{5}$ It has been documented that patients with systemic diseases such as uncontrolled type 2 diabetes mellitus, hypertension and cardiovascular disease ${ }^{6}$ are at higher risk of contracting the SARSCov-2 infection and are also afflicted more by its complications.

In this connection, mucormycosis a fungal disease caused by genera Mucorales, Absidia and Rhizopus ${ }^{7}$ has been found to occur as a co-infection in the COVID-19 illness and has been found to affect patients with uncontrolled diabetes mellitus due to their immunocompromised state. ${ }^{8}$ It is to be noted that mucormycosis also affects non diabetic individuals. When the condition affects the head and neck region, it has been termed rhinocerebral mucormycosis. ${ }^{9}$ When the maxilla is affected the lesion is termed
${ }^{1}$ Department of Dentistry, Orange Clinics, Chennai, Tamil Nadu, India

${ }^{2}$ Apollo Dental, Chennai, Tamil Nadu, India

${ }^{3}$ Department of Community Medicine, Sri Muthukumaran Medical College and Research Institute, Chennai, Tamil Nadu, India

${ }^{4}$ Department of Dentistry, Tagore Dental College and Hospital, Rathinamangalam, Tamil Nadu, India

${ }^{5,6}$ Department of Oral Pathology and Microbiology, Sri Venkateswara Dental College and Hospital, Thalambur, Chennai, Tamil Nadu, India

${ }^{7}$ Department of Maxillofacial Surgery and Diagnostic Sciences, Division of Oral Pathology, College of Dentistry, Jazan University, Kingdom of Saudi Arabia

Corresponding Author: Shankargouda Patil, Department of Maxillofacial Surgery and Diagnostic Sciences, Division of Oral Pathology, College of Dentistry, Jazan University, Kingdom of Saudi Arabia, Phone: +966507633755, e-mail: dr.ravipatil@gmail.com

How to cite this article: Arafat Y, Kumar YN, Bharani K, et al. COVID19-related Mucormycotic Osteomyelitis of the Maxilla: A Case Report of Findings, Surgical Management and Post-surgical Rehabilitation. World J Dent 2021;12(5):423-426.

Source of support: Nil

Conflict of interest: None

as maxillary mucormycosis. The fungi implicated in this condition gain entry into the human host through the nasal cavity and infiltrate the orbit, paranasal sinuses and brain. ${ }^{9}$ The initial dental manifestations of the condition include odontalgia and mobility of 
teeth. ${ }^{10}$ The events that occur following mucormycotic infiltration into the bones lead to osteomyelitis characterized by pus formation and necrosis finally leading to morbidity and in some cases even leading to death. ${ }^{11}$ It has been found that the mandible and maxilla could be affected by mucormycosis. ${ }^{12}$ However several patients post COVID-19 have manifested with maxillary and rhino-orbital mucormycosis. ${ }^{13}$ Cases of mucormycosis following COVID-19 illness have been reported in India only from the beginning of 2021. However we encountered a case of mucormycotic osteomyelitis affecting the left maxilla of 57-year-old non-diabetic female patients in a post COVID case which occurred as early as 1 year ago in the first COVID-19 wave in India and herewith present the case details and complete management of the case.

\section{Case Description}

A 57-year-old female patient reported to our maxillofacial clinic in August 2020 with the chief complaint of pain, swelling and pus discharge from left maxillary anterior teeth region. She also complained of a blocked left nostril. She was initially addressed by a general dental practitioner and was prescribed antibiotics and non-steroidal anti-inflammatory drugs which did not cause any significant improvement. The patient felt that her complaints were worsening with the time and hence approached a hospital set up for management. On clinical examination the patient was well oriented apyretic and stable. Her vital signs were normal. Intraoral examination revealed the presence of abscesses in the attached gingiva in relation to tooth numbers 13, 12, 11, 22, 23, 24 and 25 (Fig. 1A). A deep horizontal cleft was observed in the mucogingival junction in relation to 23 discharging pus. Computed tomography (CT) imaging revealed a diffuse radiolucency extending from the alveolar ridge of the upper anterior teeth to the left maxillary sinus, breaking the floor of the sinus (Figs $1 \mathrm{~B}$ and $\mathrm{D}$ ). The extent of the lesion was assessed by observation of the CT images.
Upon elucidating her medical history, it was found that she tested COVID-19 positive 3 weeks ago and underwent treatment for the same in a hospital setup. Upon eliciting her COVID-19 history, it was found that she suffered from fever, body pain and arthralgia for 3 days. She did not have any significant complaints associated with breathing. Her computed tomographic image of the lung was interpreted by the radiologist as normal as she had a CO RADS score of 2 . She did not suffer from any other medical disorders and did not have any comorbidity such as diabetes mellitus type 2 , hypertension or cardiovascular issues. She was treated by the COVID care team of the hospital with an initial oral dose of methyl prednisolone $8 \mathrm{mg}$ tapered after 1 week to a 4 mg dose, maintained for 2 more weeks. In addition to this, she was managed with daily dose of Vitamin C $500 \mathrm{mg}$, Vitamin D $2000 \mathrm{IU}$ and Asprin $75 \mathrm{mg}$ for anticoagulant effects for a time period of 3 weeks.

Following complete analysis of the case details and past medical history a provisional diagnosis of mucormycosis of left maxilla was made while the differential diagnosis included osteomyelitis following odontogenic infection. An incisional biopsy was planned and performed under local anesthesia from the lesion in relation to 23 and the tooth number 23 was extracted as it was causing discomfort to the patient. Histopathological reporting by a trained clinical pathologist revealed intense inflammatory infiltrate in the tissue section comprising predominantly of eosinophils and the presence of vasculitis. Fungal hyphae branching in right and obtuse angles could be observed in the epithelium and connective tissue regions surrounded by necrotic debris. Based on the observations a final diagnosis of mucormycosis was made (Figs 2A and D). The patient was managed both medically and surgically by our team. Surgical management included partial left maxillectomy involving the extraction of all teeth in the second quadrant in addition to teeth numbers 11,12 and 13 (Figs $1 C$ and D). The lesion was thoroughly debrided and excised carefully along with the maxillectomy procedure (Fig. 2A). Suturing was performed and
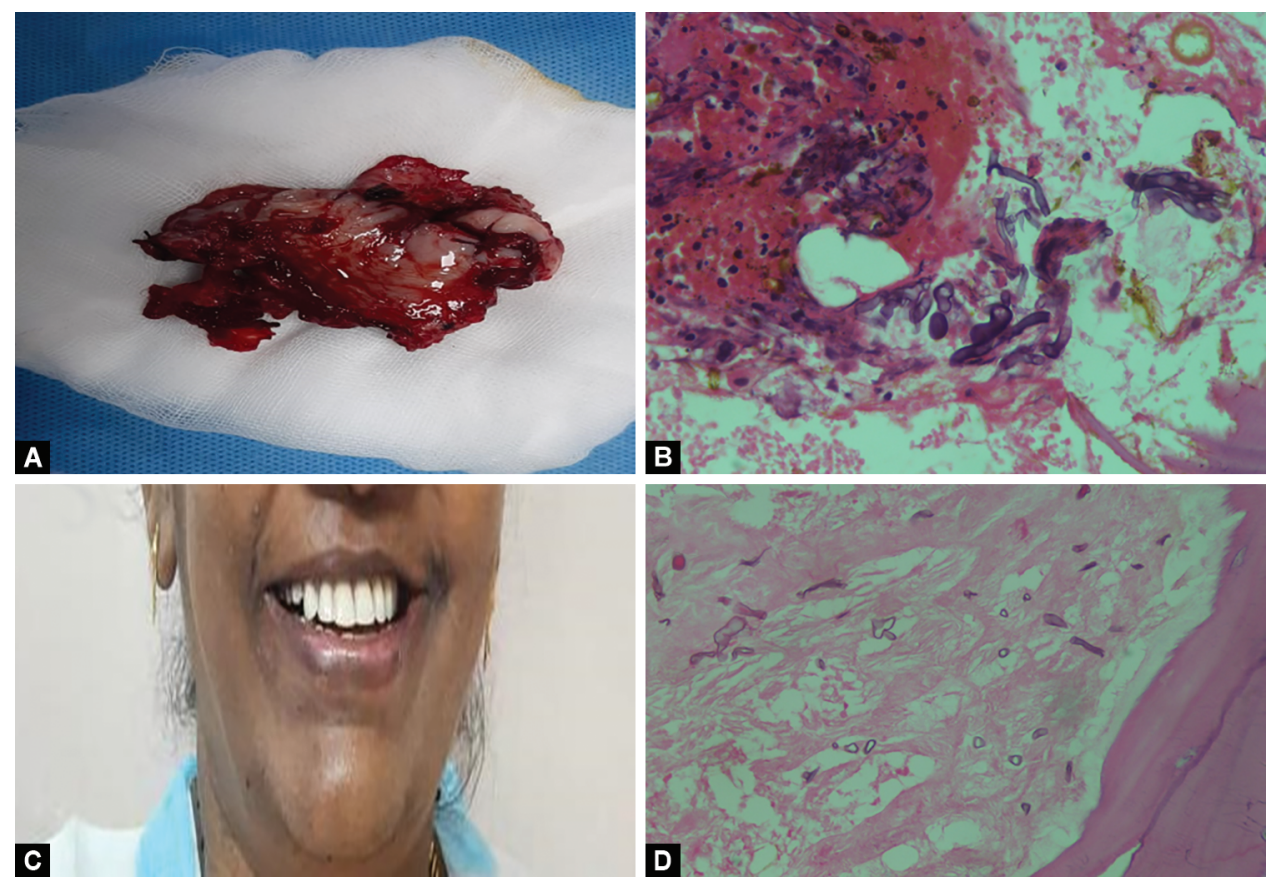

Figs $1 \mathrm{~A}$ to D: (A) Clinical preoperative intraoral photograph revealing multiple abscesses in relation to attached gingiva of the upper anterior region; (B) Computed tomography of the patient revealing multiple images of the lesion; (C) Intraoperative view of the lesion upon extracting involved teeth; (D) Intraoperative view of the lesion upon complete debridement 

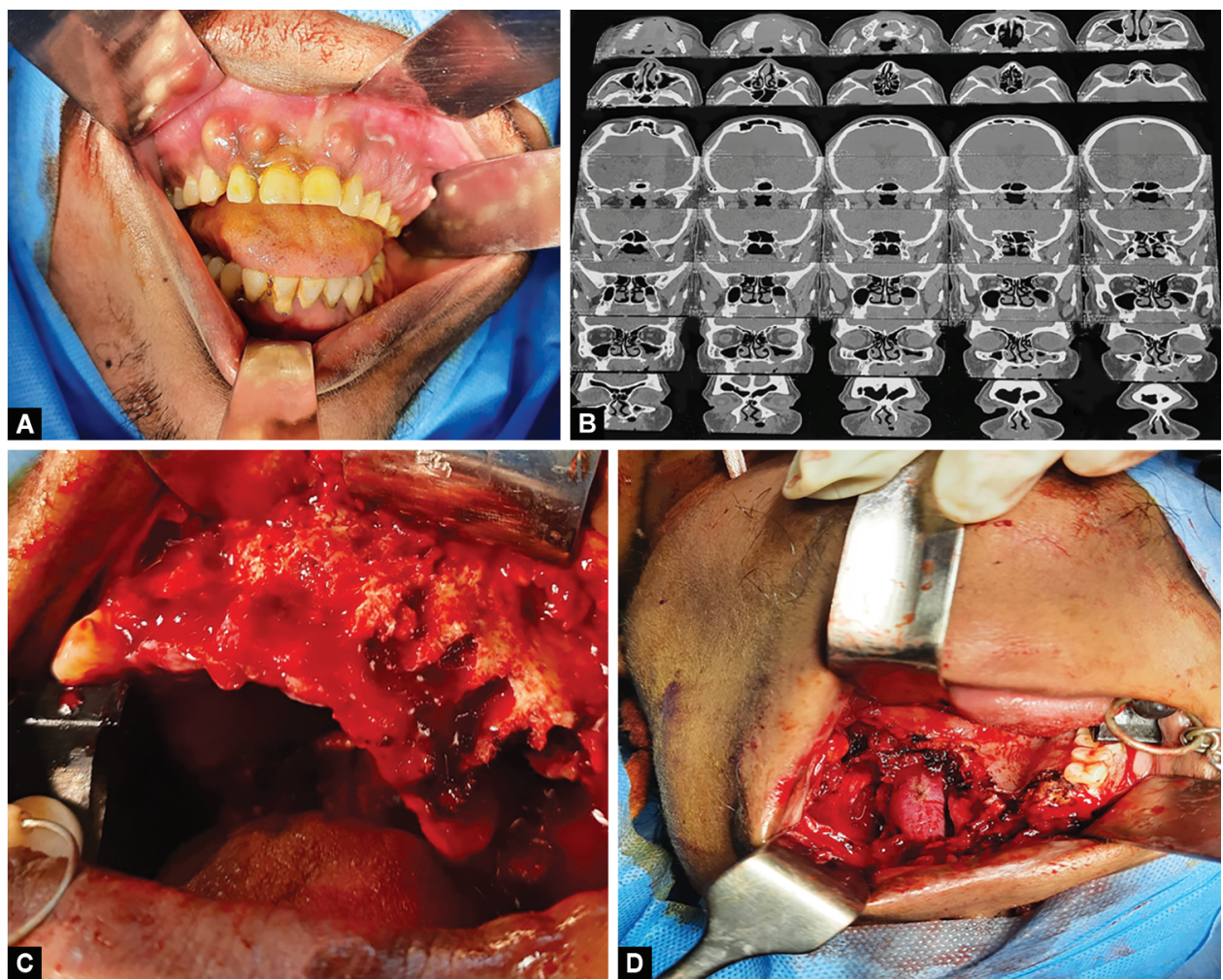

Figs 2A to D: (A) Photograph demonstrating the macroscopic characteristics of the excised lesion; (B) Histopathological image of the incisional biopsy revealing intense inflammatory infiltrate in the tissue section comprising predominantly of eosinophils and the presence of vasculitis and fungal hyphae in the epithelium and connective tissue $(40 \mathrm{X}) ;(\mathrm{C})$ Clinical photograph of the patient after postoperative prosthetic rehabilitation with obturator; (D) Histopathological image of the incisional biopsy revealing multiple fungal hyphae in the connective tissue (40X)

the patient was kept under observation for 1 week following which she was discharged. Medical management comprised of the antifungal medication amphotericin B liposomal form $(2.5$ $\mathrm{mg} / \mathrm{kg}$ body weight) slow infusion for 10-12 hours for 5 days preoperatively and postoperatively for 2 weeks, posaconazole $300 \mathrm{mg}$ tablets once daily for 28 days. The patient was prescribed paracetamol $500 \mathrm{mg}$ tablets to be take thrice a day for a week for pain management. Betadine mouthwash composed of povidone iodine diluted in warm saline in a 1:1 ratio was prescribed for rinsing 3 times a day after meals for 15 days postoperatively. Adjunctive management included a multivitamin capsule for once a day for 15 days. A postoperative review was performed after 1 week and prosthetic rehabilitation was done with an obturator for functional and esthetic concerns by a trained maxillofacial prosthodontist after 1 month (Fig. 2C).

\section{Discussion}

The SARS-CoV-2 infection has caused significant concern to humanity in the recent times. Ever since the disease has been upgraded as a pandemic by the World Health Organization, there have been concerns in both developed and developing nations. Lockdown has been imposed in many countries world over to curtail disease spread. In addition to these key practices like social distancing, wearing of masks and use of sanitizers has been diligently practiced by human populations. A vaccination drive is operating in many parts of the globe to immunize as many humans as possible to tackle the disease scourge. It is in this scenario of sincere human attempts that the deadly viral disease is still persisting and creating havoc. The occurrence of co-infections along with the COVID-19 illness increases the morbidity of the condition leading to death in many cases.

It is in this connection that osteomyelitis caused by mucormycotic infection has been studied as a COVID-19 associated manifestation. Mucormycosis is a fungal infection caused by species of fungi termed mucor, absidia and Rhizopus. These genera belong to the zygomycetes family and are known to cause infections in the immunocompromised host. Uncontrolled diabetes mellitus type 2 has been reported to be a significant risk factor for mucormycosis. The pathogenesis of the condition involves fungal entry into the host through an ambient portal such as the nose and paranasal cavities following which fungal colonization and replication occurs. The increased availability of iron in the tissues which occurs in conditions like diabetes mellitus serves as a risk factor for fungal colonization. The colonization is followed by a series of events leading to angioinvasion and extensive necrosis of underlying tissues including the bone causing pus formation and resulting in necrotic osteomyelitis. ${ }^{14}$

A case of osteomyelitis in the left maxilla has been documented by us in the present report. Our report documents the occurrence of mucormycosis in a non-diabetic patient following COVID-19 illness. With regard to the country based prevalence we have reported the present case of mucormycosis from India which has reported $72 \%$ of the share of the global mucormycosis cases. ${ }^{13}$ The interesting finding in our study is the fact that the case in concern being documented reported to our maxillofacial clinic in August 2020 
which was regarded the first wave of COVID-19 affliction in India. In fact, many of the mucormycosis cases associated with COVID-19 are being reported only in the current second wave of disease affliction. A recently published systematic review has reported that out of 101 cases of mucormycosis reported in the world 82 were from India and have affected people with active COVID-19 illness or those recovered from COVID-19. It was also found that the predominant number of cases afflicted diabetic patients and those subjects who underwent steroid therapy. ${ }^{15}$ The authors of the above review blame the unholy trinity of diabetes mellitus, COVID-19 and associated steroid use as the risk factors for mucormycosis. ${ }^{15}$

With regard to our case we found 2 of the 3 risk factors mentioned above, namely COVID-19 and steroid use for management of the same. Our patient in concern was a 57-yearold female, which is an important finding as this disease has been found to affect males with more predilection compared to females who reported only $20 \%$ prevalence compared to males reporting an $80 \%$ prevalence rate. ${ }^{15}$ Patients without diabetes mellitus also fell under the low prevalence category (20\%) as observed in the present case. With regard to the anatomical area of affliction we reported the disease in the left maxilla which is a prevalent area for disease occurrence as previously documented. ${ }^{15}$

With regard to the management of the case we followed both medical and surgical management of the patient according to the ESCMID/ECMM 2019 guidelines. ${ }^{16}$ Medical management included the use of Amphotericin B and Posoconazole which are important salvage drugs for the management of mucormycosis. ${ }^{17}$ Surgical management included left partial maxillectomy followed by complete debridement of the lesion and extraction of all the teeth in the left maxilla and teeth numbers 11, 12, 13 in the right maxilla. In addition to systemic drug therapy, we recommended a mouthwash preparation containing povidone iodine as is followed for the management of many other oral lesions. We followed up the case after review and suture removal and fabricated an obturator for the patient for esthetic and functional use. The patient is presently being followed up and has not reported of any significant morbidity.

A significant observation made by our surgical team is the fact that mucormycosis begins as a clinical condition after the fungal spores gain entry into the nose and start to colonize the maxillary antrum. From therein, the lesion can progress to involve the eyes or the brain or remain in the maxillary antrum causing osteomyelitis. Hence the maxillary antrum could be considered a vital reservoir of the pathogen. Hence we hypothesize that a comprehensive management of mucormycosis of the maxilla should focus on maxillectomy which would naturally break the antral floor and thereby establish communication with the oral cavity. In this situation, ease of drainage of exudate from the maxillary sinus would be facilitated by dependency and effect of gravity. In addition, frequent mouth-rinsing would also augment drainage from the lesion improving the case prognosis.

Our observation of maxillary osteomyelitis secondary to mucormycosis in the first COVID wave in India makes our case extremely rare and important as these highlights that COVID-19 complications were significant but under-evaluated in the first wave of the disease. Moreover our hypothesis regarding the surgical technique makes this case interesting and has a potential takehome message for the reader.

\section{References}

1. Cucinotta D, Vanelli M. WHO declares COVID-19 a pandemic. Acta Biomed 2020;91(1):157-160. DOI: 10.23750/abm.v91i1.9397.

2. Walls $A C$, Park $Y-J$, Tortorici MA, et al. Structure, function, and antigenicity of the SARS-CoV-2 spike glycoprotein. Cell 2020;181(2):281-292.e6. DOI: 10.1016/j.cell.2020.02.058.

3. Yang X, Yu Y, Xu J, et al. Clinical course and outcomes of critically ill patients with SARS-CoV-2 pneumonia in Wuhan, China: a singlecentered, retrospective, observational study. Lancet Respir Med 2020;8(5):475-481. DOI: 10.1016/S2213-2600(20)30079-5.

4. Mishra SK, Tripathi T. One year update on the COVID-19 pandemic: where are we now? Acta Trop 2021;214:105778. DOI: 10.1016/j. actatropica.2020.105778.

5. Chakraborty C, Sharma AR, Bhattacharya M, et al. The current second wave and COVID-19 vaccination status in India. Brain Behav Immun 2021;96:1-4. DOI: 10.1016/j.bbi.2021.05.018.

6. Gao Y, Ding M, Dong $X$, et al. Risk factors for severe and critically ill COVID-19 patients: A review. Allergy 2021;76(2):428-455. DOI: 10.1111/ all.14657.

7. Ribes JA, Vanover-Sams CL, Baker DJ. Zygomycetes in human disease. Clin Microbiol Rev [Internet] 2000;13(2):236-301. Available from: https://journals.asm.org/doi/10.1128/CMR.13.2.236.

8. Banerjee M, Pal R, Bhadada SK. Intercepting the deadly trinity of mucormycosis, diabetes and COVID-19 in India. Postgrad Med J [Internet] 2021. DOI: 10.1136/postgradmedj-2021-140537postgrad medj-2021-140537. Available from: https://pmj.bmj.com/lookup/ doi/10.1136/postgradmedj-2021-140537.

9. Mallis A, Mastronikolis SN, Naxakis SS, et al. Rhinocerebral mucormycosis: an update. Eur Rev Med Pharmacol Sci 2010;14(11):987992.

10. Doni $B$, Thotappa L, Peerapur B, et al. Sequence of oral manifestations in rhino-maxillary mucormycosis. Indian J Dent Res [Internet] 2011;22(2):331. DOI: 10.4103/0970-9290.84313Available from: http:// www.ijdr.in/text.asp?2011/22/2/331/84313.

11. Selvamani $M$, Donoghue $M$, Bharani $S$, et al. Mucormycosis causing maxillary osteomyelitis. J Nat Sci Biol Med [Internet] 2015;6(2):456. DOI: 10.4103/0976-9668.160039Available from: http://www.jnsbm. org/text.asp?2015/6/2/456/160039.

12. Kwak E-J, Kim D-J, Nam W, et al. Mucormycosis in the jaw: a report of 2 cases and literature review. Oral Health Prev Dent 2020;18(1):10111016. DOI: 10.3290/j.ohpd.a45522.

13. Pal R, Singh B, Bhadada SK, et al. COVID-19-associated mucormycosis: an updated systematic review of literature. Mycoses [Internet] 2021. myc.13338. Available from: https://onlinelibrary.wiley.com/ doi/10.1111/myc.13338.

14. Ibrahim AS, Kontoyiannis DP. Update on mucormycosis pathogenesis. Curr Opin Infect Dis [Internet] 2013;26(6):508-515. DOI: 10.1097/ QCO.0000000000000008Available from: http://journals.Iww. com/00001432-201312000-00004.

15. Singh AK, Singh R, Joshi SR, et al. Mucormycosis in COVID-19: a systematic review of cases reported world wide and in India. Diabetes Metab Syndr Clin Res Rev [Internet] 2021;15(4):102146. DOI: 10.1016/j. dsx.2021.05.019Available from: https://linkinghub.elsevier.com/ retrieve/pii/S1871402121001570.

16. Liang Y, Chen X, Wang J, et al. Oral posaconazole and bronchoscopy as a treatment for pulmonary mucormycosis in pediatric acute lymphoblastic leukemia patient. Medicine (Baltimore) [Internet] 2021;100(6):e24630. Available from: https://journals.lww.com/10.1097/ MD.0000000000024630.

17. van Burik J-AH, Hare RS, Solomon HF, et al. Posaconazole is effective as salvage therapy in zygomycosis: a retrospective summary of 91 cases. Clin Infect Dis an Off Publ Infect Dis Soc Am 2006;42(7):e61-e65. DOI: $10.1086 / 500212$. 